\title{
胰岛素介体的产生与诱导的激素活性的关系*
}

\author{
乐云张世荣幸兴球 \\ （中国科学院动物研究所内分必室,北京）
}

最近对勝岛素作用机理的研究指出, 胰岛素与它的靶细胞受体结合后, 激活细胞膜上的蛋 白水解酶 ${ }^{[1,2]}$,或磷脂酶 $\mathrm{C}^{[3]}$ 而导致膜上某种糖蛋白或磷脂水解, 产生多肽样 ${ }^{[1,4,5]}$ 或磷脂样 ${ }^{[6,7]}$ 的 化学介体. 它们能在无细胞系统中模拟胰岛素的各种作用, 如, 调节胰岛素敏感的酶的活性, 以及在完整细胞中模拟胰岛素的一些生物功能,包括调节 cAMP 水平、腺草酸环化酶活性、抗 脂解作用和脂肪合成等 $[5,8-10]$. 这些研究结果证实了胰岛素介体可能是胰岛素的第二信使.

最近, 我们报道了两种胰岛素类似物诱导猪肝细胞膜产生胰岛索介体组分 1 与费岛素的 唀导能力存在明显的差别, 而这种差别与它们的生物活性相关 ${ }^{[1]}$. 本文为了进一步了解胰帛 紫介体的产生是与受体结合相关还是一种受体后机制，我们用具有不同生物活性和受体结合 活性的胰岛素及其类似物与猪肝细胞膜保温,比较它们诱异产生的胰岛素化学介体的关系。

\section{一、材料和 方法}

1. 材料胶原酶 (collagenase) 购自 Worthington; $\left[8{ }^{3}{ }^{3} \mathrm{H}\right] 3^{\prime}, 5^{\prime} \mathrm{cAMP}, 23 \mathrm{Ci} / \mathrm{mmol}$ 和 $\left[3^{-3} \mathrm{H}\right]$ 葡䔨糖, $5.3 \mathrm{Ci} / \mathrm{mmol}$, 购自 New England Nuclear; Dowex AG50w $\times 8$, 孔径 100-200目, 购自 Bio-Rad; 牛血清白蛋白 (BSA) 第五组分, 购自 Sigma; 人胰岛素 (HIns)、猪胰岛素 (P-Ins) 及它的三个类似物: 羧端酰胺化胰岛素 $\left(\mathrm{Ins} \mathrm{NH}_{2}\right) 、 B$ 链羧端去五 肽酰胺化涙岛素 $\left(\mathrm{DPINH}_{2}\right) 、 \mathrm{~B}$ 链羧端去七肽酰胺化胰岛素 $\left(\mathrm{DHINH}_{2}\right)$, 为中国科学院生物 化学研究所朱尚权教授馈赠。这些胰岛素及其类似物的整体活力(引起小鼠惊顸反应)和与人 胎照细胞膜受体结合活力见表 1 .

2. 方法 (1) 肝细胞膜、涙岛素化学介体游离脂肪细胞的制备及脂肪合成测定按文献 [8] 方法进行.

（2）腺苷酸环化酶（AC）活性测定按文献[10]方法进行.

\section{二、结 果}

\section{1. 费岛美及其类似物促进大白鼠脂肪细胞的脂肪合成作用比较 首先取胰岛素及其类} 似物浓度分别为 $0.187,0.56,0.93,1.86$ 和 $3.73 \mathrm{ng} / \mathrm{ml}$ 与大鼠脂肪细胞保温, 直接测定它们的 促进脂肪合成作用. 图 1 为胰岛素及其类似物促进脂肪合成作用的剂量曲线. 从图 1 可以看 出它们的促进脂肪合成作用，随浓度的增加而增大. 作用大小顺序为

$$
\mathrm{P}-\mathrm{Ins}>\mathrm{H}-\mathrm{Ins}>\mathrm{DPINH}_{2}>\mathrm{InsNH}_{2}>\mathrm{DHINH}_{2}
$$

若以猪胰岛素活力为 $100 \%$ ，则计算得人胰岛素及猪胰岛素衍生物的活力分 别为 85,65

- 国家自然科学基金资助项目. 
58 和 $44 \%$ 。这个结果与引起小鼠惊厥反应的整体活力大小基本相符, 而与受体结合活力没有 相关性（见表 1)。

表 1 几种肤岛素及其类似物的活力比较

\begin{tabular}{|c|c|c|c|}
\hline 用舟菜及其类似物 & $\begin{array}{c}\text { 受体结合活力 } \\
\text { (胎盘细跑㙞) } \\
(\%)\end{array}$ & 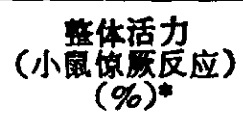 & $\begin{array}{c}\text { 促进脂助含成作用 } \\
(\%))^{2}\end{array}$ \\
\hline P-Ins & 100 & 100 & 100 \\
\hline H-Ins & 100 & 90 & $85 \pm 3.4$ \\
\hline DPINH $_{2}$ & 187.7 & 83 & $66 \pm 4.8$ \\
\hline Ins $\mathrm{NH}_{3}$ & 89 & 58 & $58 \pm 3.3$ \\
\hline $\mathrm{DHINH}_{2}$ & 9.4 & 40 & $44 \pm 5.5$ \\
\hline
\end{tabular}

- 为中国科学院生物化学研究所朱尚权数授的研究组测定结果.

**根据图 1 后 4 点计筆的平均值士方差(B. P-Ins 话力为 $100 \%$ 计算).

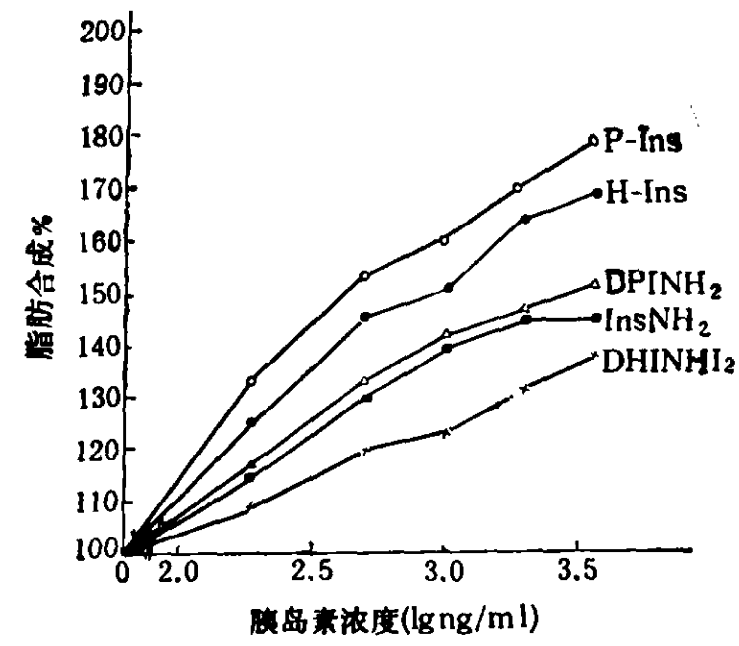

图 1 腃岀索及其类似物对脂肪细胞 合成的影响

用不同活生, 不同浓度的电泳纯 P-Ins 及其类似物 Ins $\mathrm{NH}_{2}$,

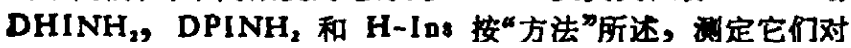
朌肪细绚脂肪合成的影响. 每一点为三次实验的平均值

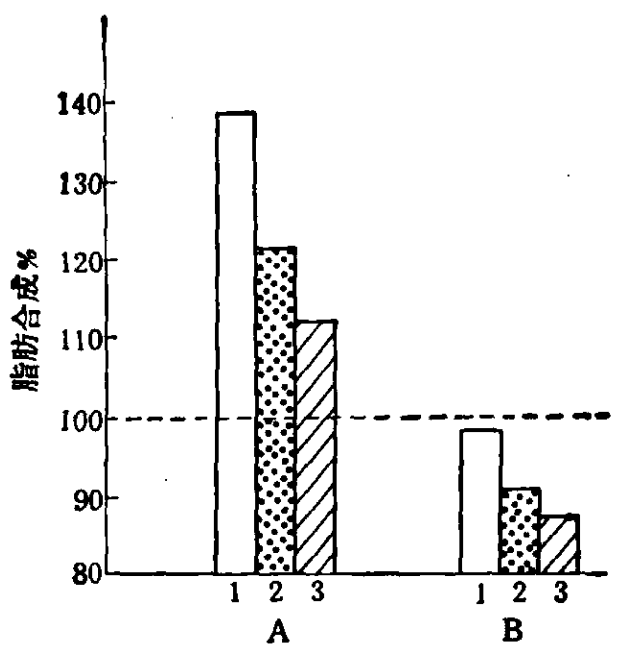

图 2 胰岛素及其类似物诱导的介体组分 1 和介 体组分 2 对大百鼠脂肪细胞脂肪合成的影响

1. Ins; 2. DPINH ; 3. DHINH ${ }_{2}$. $11.2 \mathrm{ag} / \mathrm{ml}$ 的 $108, \mathrm{DPINH}_{2}, \mathrm{DHINH}_{2}$ 与 $5 \mathrm{mg}$ 蛋白 $/ \mathrm{ml}$ 所 细胞质膜保温，所得离心上清湤浓缩三倍，按“方法” 所述层析分离，各取介体组分 1 和组分 2 各 $150 \mu 1$, 按“方法”所达，测它们对脂肪合成的促进和抑制活力 的差异. A. 介体组分 1; B. 介体组分 2. 图中每一 柱为三次实验的平均值

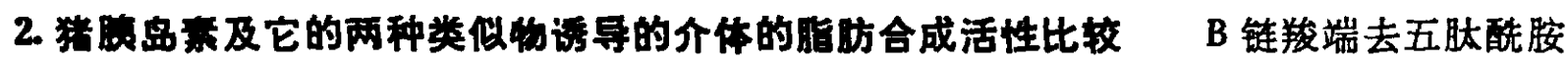
化胰息素 $\left(\mathrm{DPINH}_{2}\right)$ 具有十分高的受体结合活力, 为自然猪胰岛素的 $187.7 \%$ ，而生物活力 (促进脂肪合成作用和引起小鼠惊厥反应)却低于猪胰岛素 (66\%)，B 链羫端去七肽酰胺化费 少 $\left(\mathrm{DHINH}_{2}\right)$ 的受体结合活力极低 $(9 \%)$ 而生物活力虽然偏低, 但却大大超过其与受体结 合的活力 $(44 \%)$. 因此，我们选用这两种类似物与猪胰岛素比较诱导介体的产生,对了解胰岛 素介体的产生是取决于与受体结合抑或与受体后的反应相关将会有一定的帮助.

在另一文中我们已报道猪䐀岛素与猪肝膜保温可诱导产生促进型和抑制型两种介体一 组分 1 和组分 2. 这里, 我们按照该方法, 用 $11.2 \mathrm{ng} / \mathrm{ml}(300 \mu \mathrm{U} / \mathrm{ml})$ P-Ins, $\mathrm{DPINH}_{2}$ 和 $\mathrm{DHINH}_{2}$ 分别与猪肝细胞膜保温, 并用不加胰岛素的猪肝细胞膜保温液作对照, 离心, 上清 


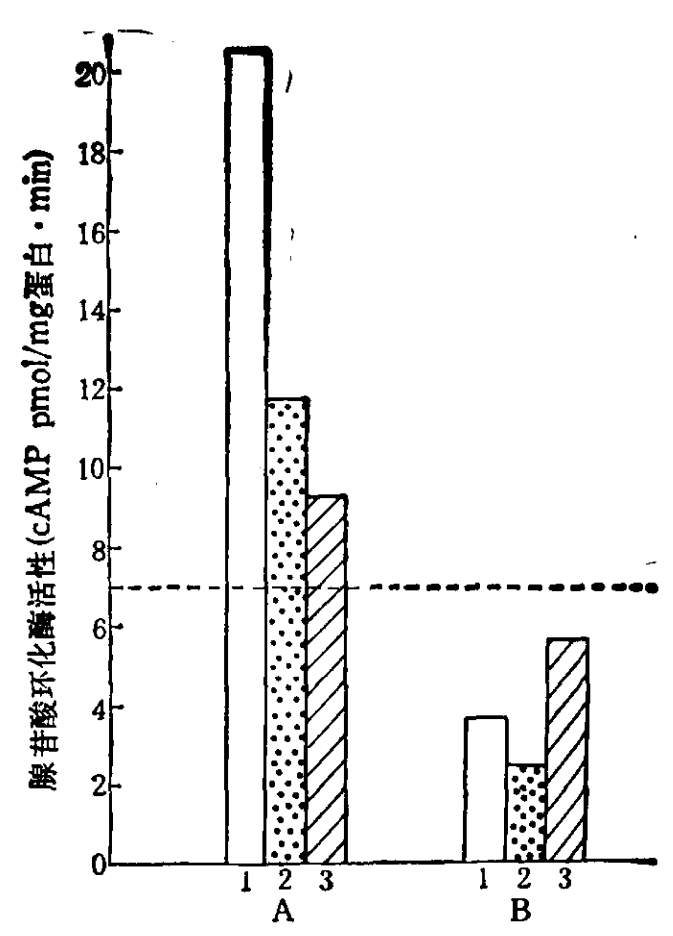

国 3 眱岛紊及其类似物的介体组分 1 和 2 对猪肝细胞膜腺莫酸环化酶活性的影响

1. Ins; 2. DPINH $;$;. DHINH 2 . 诱导和分离条件与图 2 相同.

取介体组分 1 和 2 各 $40 \mu$ l，按 “方法”所述. 测定它们对腺苷酸环

化酶活性的影响. 每一柱为四次测定的平均值.

A. 介体组分 $1 ;$ B. 介体组分 2

液经 Sephadex G-25 层析, 分别收集介体组分 1 和组分 2, 测定它们的脂肪合成作用. 图 2 表明诱导产生的介体组分 1 的促进脂肪合成作用和介体组分 2 的抑制脂肪合成作用. 以不加 腾岛素的对照组分为 $100 \%$ 计算, 则 P-Ins, $\mathrm{DPINH}_{2}$ 和 $\mathrm{DHINH}_{2}$ 诱导的介体组分 1 促进脂 肪合成作用分别为 $137.5,121.5$ 和 $111.5 \%$; 介体组分 2 的抑制脂肪合成作用分别为 99,82 和 $69 \%$.

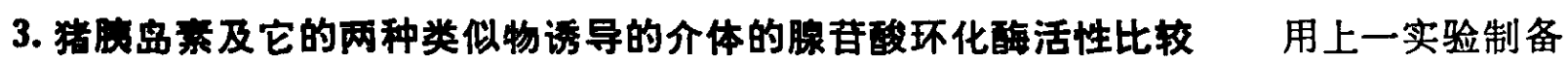
的介体组分 1 和组分 2 分别测定它们对腺苷酸环化酶活性的影响. 结果表明猪腾岛素及它的 两种类似物诱导产生的介体组分 1 有明显的促进腺苷酸环化酶活性的作用, 而介体组分 2 则 表现为抑制该酶活性的作用 (见图 3). 以不加胰岛素的对照为 $100 \%$ 计算, P-Ins, DPINH 和 $\mathrm{DHINH}_{2}$ 诱导产生的介体组分 1 对腺苷酸环化酶活性的促进作用分别为 290,169 和 $130 \%$ ，而介体组分 2 对该酶活性的抑制作用分别为 $52.6,33.3$ 和 $82.3 \%$.

\section{三、讨 论}

在文献 [12]中我们曾经报道用等量的具有不同生物活性的猪胰岛素及其两种类似物一 B 链 $\mathrm{N}$ 端加一个 $\mathrm{D}$-丙氨酸的胰岛素和 $\mathrm{B}$ 链 $\mathrm{N}$ 端去掉第一个氨基酸残基, 羧端去掉 5 个氨基酸 残基的胰岛素制备介体组分 1 , 以测定它们促进脂肪合成的活性为指标, 发现胰岛素及其类似 物的活性与诱导介体组分 1 的产量相关. 㪳工作应用猪滕岛素的另两种类似物做了进一步的 比较。它们在生物活力和受体结合活力上都有较大差别, 它们都能诱导肝细胞膜产生促进型 的介体组分 1 和抑制型的介体组分 2 . 介体组分 1 表现有明显的促进脂肪合成作用和提高腺 
某酸环化酶的活性，其活性大小表现出与诱导的胰岛素及其类似物的生物活力相关。即生物 活力高的胰岛素诱导产生的介体组分 1 活性也高, 生物活力低, 则诱导产生介体组分 1 活性也 低, 而与它们的受体结合能力无关. 这与前文的实验结果一致, 并与我们提出的假说: “介体 产生是一个受体后反应机制”相吻合。

Ins, $\mathrm{DPINH}_{2}$ 和 $\mathrm{DHINH}_{2}$ 诱导产生的介体组分 2 对脂肪合成的抑制能力则以 $\mathrm{DHINH}_{3}$ 诱导的介体组分 2 作用最强, $\mathrm{DPINH}_{2}$ 诱导的次之, Ins 诱导的最低. 这种结果并不奇怪, 因 为另一实验已证明(实验结果还末发表)用 100,200 和 $300 \mu \mathrm{U} / \mathrm{ml}$ 的 P-Ins 诱导产生的介体 组分 2 对脂肪合成的抑制作用分别为对照的 74,80 , 和 $90 \%$ 。而本实验用同样量 $11.2 \mathrm{ng} / \mathrm{ml}$ 的 Ins, $\mathrm{DPINH}_{2}$ 和 $\mathrm{DHINH}_{2}$ 实际相当于 Ins 的效价为 300,200 和 $120 \mu \mathrm{U} / \mathrm{ml}$. 因此, 不难 理解，它们诱导的介体组分 2 的活力关系为 $\mathrm{DHINH}_{2}>\mathrm{DPINH}_{2}>\mathrm{Ins}$. 这实验结果恰巧与另 一实验结果相符合, 即低生理浓度的激素诱导介体组分 2 为主, 而高于生理浓度的激素则诱导 产生介体组分 1 为主.

同样,测定介体组分 2 对腺苷酸环化酶的作用,发现 $\mathrm{DPINH}_{2}$ 诱导的介体组分 2 对该酶活 性的抑制作用最强。在另一报道中指出, 100,200 和 $300 \mu \mathrm{U} / \mathrm{ml}$ 的胰离素诱导的介体组分 2 对腺葉酸环化酶的抑制分别为对照的 3.6, 3.07 和 1.6 倍. 因而, DPINH (有效浓度 $200 \mu \mathrm{U} /$ $\mathrm{ml})$ 诱导产生的介体组分 2 对腺苦酸环化酶的抑制作用较 Ins $(300 \mu \mathrm{U} / \mathrm{ml})$ 诱导的强，是与 上述结果一致的. 但是, $\mathrm{DHINH}_{2}$ (有效浓度 $120 \mu \mathrm{U} / \mathrm{ml}$ ) 诱导的介体组分 2 对腺葉酸环化 酶活性的抑制作用很弱, 是与上述结果不一致的, 估计 $\mathrm{DHINH}_{2}$ 的生物活力太低, 影响到介 体组分 2 的产生,仍有待于进一步的实验加以证实.

从不同浓度的胰岛素 ${ }^{[11}$ 和不同活力的胰岛素和其类似物都证实胰岛素可从其靶细胞，所 细胞质膜中诱导产生两个分子量不同、生物功能相拮抗的介体. 其中,在较高浓度或活力的激 意诱导产生介体组分 1 为主, 而在较低浓度或活力较低的情况下(接近生理浓度)产生抑制作 用的介体组分 2 为主. 因此, 从不同浓度和不同活力的两组实验所得结果基本相同, 可以证明 : 在不同生理情况下由于激素浓度变化诱导靶细胞产生不同的化学介体对细胞进行平衡调节。

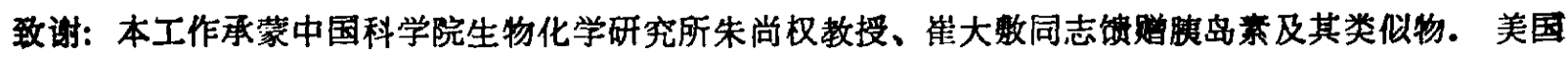
迈阿密大学医学院生化系教授 Dr. Antcro G. So 犆赠化学试剂. 姚文贞、韩锦华等同志在实验工作中热情 协助, 特此一并致谢.

\section{参考嗝}

,[ 1] Seals, J. R. \& Czech, M. P., J. Biol. Chem., 256(1980), 2894-2899.

. [2] Kono, T. \& Barham, F. W., ibid., 246(1971), 6204-6209.

[3] Houslay, M. D. et al., Trends Biochem. Sci, 11(1986), 393-394.

[ 4 ] Suzuki, S. et al., Biochem. Biophys. Res. Commun., 118(1984), 40-46.

[ 5 ] Zhang, S. R. et al., J. Biol. Chem, 258(1983), 6471-6476.

[6] Satiel, A. R. \& Cuatrecasas. P., Proc. Natl. Acad. Sci. USA, 83(1986), 5793-5797.

[ 7 ] Satiel, A. R. et al., Science, 233(1986), 967-969.

[8] 张世荣等, 中国科学, B辑, 1987,265-272.

[9] 张世荣等, 生物化学与生物物理学报, 19(1987), 79-86.

[10] Zhang, S. R. et al., Scientia Sinica (in press).

[11] Wei, T. Q. \& Zhang, S. R., Seientia Sinica, Ser. B, 30(1987), 1287-1293. 